\title{
Medical malpractice claim risk in emergency departments
}

\author{
Claudio Bianchin, ${ }^{1}$ Carolina Prevaldi, ${ }^{2}$ Matteo Corradin, ${ }^{3}$ Mario Saia ${ }^{4}$
}

${ }^{1}$ Veneto Region Local Health Unit n. 1, Department of Risk Management and Patient Safety, S. Martino Hospital, Belluno; ${ }^{2}$ Veneto Region Local Health Unit n. 4, Department of Risk Management and Patient Safety, San Donà di Piave; ${ }^{3}$ Veneto Regional Center for Patient Safety, Padova; ${ }^{4}$ Veneto Region, Local Health Unit n. 6, Padova, Italy

\begin{abstract}
Medical malpractice claims are a major problem for emergency physicians and for the health system which must be addressed in a rational and effective fashion: claim analysis seems the best way to identify risk factors and risk areas and to elaborate risk management recommendations. The Emergency Department (ED) is one of the areas at higher risk. Medical diagnoses associated with the highest number of claims are acute myocardial infarction, fractures, appendicitis, abdominal/pelvic symptoms, aortic aneurism and open wounds to fingers. The present paper emphasizes the necessity for ED emergency physicians to pay special attention when facing these health conditions and seeks to provide indications in order to reduce litigation.
\end{abstract}

\section{Introduction}

There is little doubt that medical malpractice claims, whether successful or not, are a major problem for emergency physicians and for the health system in general, which must be addressed in a rational and effective fashion. For this reason, proactive strategies must be developed aimed at reducing the risk of litigation and at making treatments less vulnerable in case of litigation. Claim

\footnotetext{
Correspondence: Claudio Bianchin, Veneto Region Local Health Unit n. 1, Department of Risk Management and Patient Safety, S. Martino Hospital, viale Europa 22, 32100 Belluno, Italy.

Tel.: +39.0437 .514233 .

E-mail: claudio.bianchin@ulss.belluno.it

Key words: Emergency departments; Claims; Malpractice; Risk management.

Contributions: all the authors contributed extensively to the work presented in this paper; CB supervised it.

Conflict of interest: the authors declare no potential conflict of interest.

Funding: none.

Received for publication: 2 November 2016.

Revision received: 22 May 2018.

Accepted for publication: 22 May 2018.

This work is licensed under a Creative Commons Attribution 4.0 License (by-nc 4.0).

(C) Copyright C. Bianchin, 2018

Licensee PAGEPress, Italy

Emergency Care Journal 2018; 14:6366

doi:10.4081/ecj.2018.6366
}

analysis of large amounts of data on malpractice claims seems the best way to identify risk factors and risk areas and to elaborate recommendations that will help prevent lawsuits or make the positions of health care providers more defensible. ${ }^{1}$

Understanding which diagnoses and clinical conditions are liable to lead to lawsuits, and for what reason, will help doctors and health care organizations implement efficient risk-containing strategies. Priorities in research on medical malpractice claims are frequency, average amount paid (average indemnity) ratio of paid claims to total claims (indemnity payout rate).

\section{Malpractice claims analysis in the emergency department}

The Emergency Department (ED) is notoriously one of the areas at higher risk. EDs are unique settings, which, due to a combination of complex factors, are particularly vulnerable to medical errors. Doctors in the ED must take quick decisions concerning patients in critical conditions, often based on incomplete or incorrect information, in an environment in which they are frequently interrupted and distracted. Medical errors in ED are associated with a high number of malpractice claims, which have a significant financial impact.

An important source for information is the data collected and systematically analyzed by the Physician Insurers Association of America (PIAA), an association of insurance companies which totally cover about $60 \%$ of doctors practicing in the United States. PIAA uses this data to assist health organizations in containing the risk of malpractice claims.

PIAA data for 1985-2007 contains more than 11,500 claims associated with EDs. ${ }^{2}$ Of these, $31 \%$ resulted in payments. The average compensation was $\$ 188,000$. In about $20 \%$ of cases ED doctors were involved in the lawsuit; the rest of the cases concerned other professionals responsible for ED care or consulting, such as orthopedic surgeons, general surgeons, internists, radiologists, cardiologists, etc.

Two thirds of these claims were settled out of court without payment. Also most of paid claims were settled out of court. Only a minority was decided in court and the verdicts were largely in favor of doctors. In total, $70 \%$ of the cases ended without any payment. However, they still led to considerable legal expenses. Lawsuits settled out of court were considerably less expensive $(\$ 175,000)$ than those settled in court in favor of the patient $(\$ 393,000)$, also due to lower legal expenses.

In general, over the years there was a small decrease in the total number of closed claims and paid claims, whereas both the average indemnity paid and average legal expenses per case significantly increased.

The most common category of errors are diagnostic errors $(37 \%)$, consisting in wrong, delayed or missed diagnosis, followed 
by inadequate/missed/delayed execution of a procedure $(24 \%)$. The first category includes almost half of all compensations paid. Other common error categories include inadequate monitoring (7\%) or pharmacological therapy, missed/delayed consulting and omitted/late hospitalization. In $18 \%$ of the cases no error was identified; however, in part of these cases compensation was paid anyway though average amounts were lower.

Medical diagnoses associated with the highest number of claims are acute myocardial infarction (AMI), along with chest pain, not further defined $(9 \%)$, fracture $(6 \%)$ and appendicitis (2\%). The top ten also include symptoms involving abdomen/pelvis (4\%), injury to multiple parts of the body (3\%), aortic aneurism $(2 \%)$ and open wounds to fingers $(2 \%)$.

This distribution is basically confirmed by the PIAA report of 2011 and does not differ significantly from that of other studies on high-risk diagnoses in ED, although in some cases it is difficult to compare studies due to different classifications adopted. ${ }^{3,4}$

As for the severity of injury, death accounted for $36 \%$ of all closed claims, $40 \%$ of all paid claims and about $50 \%$ of total amount paid. The most common diagnosis associated with cases involving death were AMI and chest pain, followed by aortic aneurism, symptoms involving abdomen/pelvis and pulmonary embolism. Together with death, three damage categories (significant permanent, major permanent and severe) accounted for $80 \%$ of the total indemnity paid.

Most claims concerned missed or delayed diagnoses more than treatment errors. Claims concerning diagnostic errors were also the more frequently compensated ones. Further research has sought to identify with greater precision those aspects of the diagnostic process most frequently involved in legal litigation associated with ED. In one study on 122 settled cases involving ED, collected by 4 insurance companies, ${ }^{5}$ the main errors in the diagnostic process concerned prescription (58\%), imprecise interpretation of appropriate exams (37\%), incomplete medical history or physical examination $(42 \%)$ and timeliness of the request for necessary consultation $(33 \%)$.

Among the most common errors due to missing prescription of appropriate diagnostic exams, the most frequent involved X-rays (22\%), followed by computed tomography (CT) scans (17\%), cardiac enzyme level (15\%), ultrasound scans (11\%). Also interpretation errors involved mostly X-rays.

Factors that contributed the most to missed diagnoses were cognitive factors, which were present in $96 \%$ of cases. These included judgment errors, lack of knowledge or technical skill, attention or memory errors. Also common were patient-relatedfactors $(34 \%)$, such as insufficient compliance, atypical clinical presentation or complicated medical histories. Other relevant factors were inadequate supervision $(30 \%)$, inadequate handoffs (24\%), and excessive workload (23\%). Often, the omission of the diagnosis was the result of several errors, factors or doctors.

In Veneto Region (Italy), data about claims are systematically included by health authorities in a national database called SIMES. Presently, we have data for the 2009-2013 period. Unfortunately, the usefulness of the data is limited, since it is not always possible to identify the type of incident and the cause because the information in the pertinent fields is insufficient. The database contains 307 cases of injury and 56 cases of death involving ED. In this dataset too, diagnostic errors are prevalent, both for injury (207; $67 \%)$ and for deaths $(37 ; 71 \%)$. The remaining errors are mostly associated with therapy, while other types of errors are marginal (e.g. fall, infection). The number of non-classified cases is high to the point of making the quantitative data unreliable, but results are similar to other datasets. Specifically, the analysis of the claims shows that there is a significant group of injuries involving fractures (limbs and vertebrae), tendon lesions, and wounds (e.g. infections, foreign bodies, scars), while deaths are typically due to cerebral hemorrhage, acute abdominal complications (e.g. peritonitis, bowel infarction, perforated viscus), heart attack, ruptured aortic aneurism and pulmonary embolism.

\section{Chest pain and myocardial infarction}

AMI is the diagnosis with the highest association with death, the highest paid-to-close ratio (42\%) and the highest average indemnity paid. Heart attacks are often the cause of litigation involving missed diagnosis. The study of patients discharged from the ED with an acute coronary syndrome (ACS) which then progresses in AMI is of great interest. Less frequent are the cases concerning issues with treatments, request for advice or patient transfer. These concern mostly patients with atypical symptoms. There are a large number of cases involving young patients, women $(<55)$ and patients without documented history of coronary artery disease or without ischemic alterations in the first ECG. Older patients and diabetics often have potentially misleading symptoms. Doctors in the ED need to be very careful with atypical symptoms that could derive from an ACS. Retrospectively, the decision to discharge a patient might easily be judged imprudent.

The most common claims concern inadequate documentation. Description of the features and of factors causing and reducing chest pain, of the associated symptoms, or a complete anamnesis of cardiovascular risk factors are often lacking. Also absent are explicit instructions at discharge for the re-evaluation and follow up of pain of undetermined nature. Other vulnerabilities concern omitted comparison with previous ECG and the over-reliance of tests (e.g. a single determination of cardiac enzymes or a negative ECG). It is important for the documentation to be integrated by brief notes that reflect the decisional process, the risk-benefit analysis (e.g. the absence of contraindications for thrombolysis) and the discussion with the patient (or family members), though not necessarily in terms of written consensus.

When the problems of the patient exceed the capacity of the ED doctor or of the service, decisions must be shared with more experienced colleagues, consultants or other hospitals that could offer more adequate diagnostic or therapeutic interventions. Emergency treatment should nonetheless be ensured while waiting for consultation or transfer.

\section{Bone fractures}

Another frequent cause of litigation, albeit with lower average indemnity, is the missed diagnosis of fractures or dislocation and, to a lesser degree, of lesions to tendons, ligaments or nerves. ${ }^{6}$

Also significant are complications resulting from wounds, such as infection or presence of foreign bodies. ${ }^{7,8}$ Diagnosis of fractures are most commonly missed in the limbs: phalanx, metacarpus and metatarsus, scaphoid, calcaneus, malleolus (mostly lateral), distal radius (e.g. epiphysis and styloid), but also elbow (e.g. radial head), knee (e.g. tibial plateau); hip and shoulder (e.g. clavicle); other areas at risk are the vertebrae (lumbar and dorsal), the cranium (e.g. zygoma) and greenstick fractures. The origin of the error is normally ascribed to an incorrect interpretation of the $\mathrm{X}$-rays by the radiologist (or orthopedic surgeon), but sometimes it is ascribed to decisions by ED doctors, in the form of omitted prescriptions of X-rays, wrong area indication or insufficient information given to a consultant, sometimes as a consequence of an inaccurate clinical exam. Risk factors include difficulty in localizing injury, superficial clinical investigation, underestimation of trauma, distracting presence of another injury on the same limb or on 
the contralateral limb.

Errors are typically discovered later, due to persisting symptoms, with new or more accurate exams, by the same ED doctor who discharged the patient or by a different health structure to which the patient had turned or during the examination of other contextual injuries.

The delay in the diagnosis may lead to long-term consequences (e.g. tendon injuries, mal-union), increased surgery risks (e.g. femur fracture), or simply be the cause of a longer period of pain and discomfort. All these may lead to malpractice claims.

ED doctors, after an exhaustive medical history and careful physical exam of the patient, must decide if X-rays can be reasonably deemed not necessary, or must provide the radiologist all the information necessary to precisely identify the area to be examined and decide the imaging technique, as well as ask for repetition or integration in case of inadequate quality. At the very least, the patient should be followed up promptly if symptoms persist; if the delay is brief, it will be possible to simply apologize to the patient and treat the injury without any further complication.

\section{Abdominal pain}

In the category of abdominal pain, the missed/delayed diagnosis of appendicitis is the main cause of malpractice claims against ED doctors. In most cases we are dealing with complicated appendicitis: perforated, evolved into peritonitis, requiring laparotomy, abscess drainage or leading to post-surgery complications. The analysis of large datasets of cases of acute appendicitis leading to malpractice claims shows an association with certain characteristics of the clinical from which recommendations for ED doctors can be derived. In most cases, the initial presentation was not a normal one, symptoms were less obvious and the general picture less serious, suggesting that attention should be paid to atypical forms. In particular, in patients with uncertain gastro-intestinal or abdominal symptoms, documental evidence is critical and must include an exhaustive clinical history, and a complete physical examination, preferably including rectal examination; this well help prove due diligence in reasonably excluding appendicitis as a cause. Another risk factor is the administration of narcotic pain medications followed by discharge. Narcotic pain medications should be considered only for patients for whom observation, hospitalization or surgery consultancy is planned. Another recurring case is the wrong diagnosis of gastroenteritis, notwithstanding the lack of evidence; in these cases it is important to rigidly follow a protocol to avoid discharging patients that might develop appendicitis with complications. Finally, another frequent issue is the lack of clear instructions for the follow-up at discharge. For patients discharged with uncertain abdominal pain or symptoms, a check must be planned within the next 12 hours or in case certain symptoms emerge or do not decrease within a certain period. Lawyers commonly emphasize that if the exams do not convincingly exclude appendicitis, the doctor should ask for a close follow-up. It must be said that, though the use of expert systems and reliable score systems helps reduce both perforations and negative laparotomies, the decision whether to subject the patient to immediate surgery, simple observation or discharge remains very difficult.

These recommendations can be useful also for other causes of abdominal pain such as diverticulitis, cholecystitis, perforation of the viscera, ectopic pregnancy.

\section{Neurological problems}

In the ED, the missed diagnosis of critical neurological prob- lems is also common, problems consisting mostly in subarachnoid hemorrhages and strokes/transient ischemic attack (TIA). These cases are not very frequent but their financial impact is high due to the serious consequences. The most typical errors include omitted request for exams (CTs mostly but also magnetic resonance imaging), omitted request for hospitalization (especially for TIAs), omitted request for specialist advice, inadequate follow-up instructions, inadequate history or physical examination.

Headache is a common complaint and it may be difficult to discriminate primary forms from those that might be warnings of serious conditions, requiring appropriate and timely exams and treatment. Patients with spontaneous subarachnoid hemorrhage do not always exhibit typical symptoms, such as intense headache of sudden onset. Also, their neurological history and neurological examination results are often normal, so that in more than $25 \%$ of cases the initial diagnosis fails to detect the condition. Therefore, when confronted with a normal headache it is important to maintain a critical and open mind, scrupulously documenting the anamnesis (in particular a previous history of headache), the physical examination (e.g. meningism, fundus, cranial nerves, etc.) and possible changes over time. In case of discharge, the need for observation and a future check-up at the ED must be explained clearly and in detail, verifying that the patient or the caregiver has understood, possibly with the use of preprinted instructions.

Strokes do not normally entail the same diagnostic problems - though sometimes it may be difficult to diagnose them in the case of young subjects or strokes of the posterior circulation or the cerebellum - but it has became a typical medico-legal problem after the advent of thrombolityc therapy. ${ }^{9}$ Standards are still not well defined; however, it is important to adopt a valid specific procedure and produce documentary evidence that one has followed it. Although, after the advent of thrombolytics and mechanical revascularization ${ }^{9}$ standards are still evolving, it is important to follow a protocol and to produce documentary evidence that this has been done. It is also useful to use appropriate forms both for consensus or denial of thrombolytic therapy.

\section{Pulmonary embolism}

Typical claims against ED physician also include the failed diagnosis of pulmonary embolism; ${ }^{10}$ the possibility of this condition should always be taken into consideration in any acute cardiovascular clinical presentation. Since anticoagulant therapy has been shown to reduce mortality, injury or death following a failed diagnosis can be easily considered the result of negligence. Unfortunately, in about $20 \%$ of the cases, the typical triad (haemoptysis, dyspnea, and chest pain) is absent, and no single aspect of the physical examination can ensure a reliable diagnosis. The frequent simultaneous presence of other cardiovascular conditions (congestive heart failure, coronary diseases or chronic obstructive pulmonary disease, also characterized by chest pain and shortness of breath, can make the diagnosis of pulmonary embolism difficult. Another source of confusion, in differential diagnosis, is dyspnea in pregnant women, in which the risk is five times higher. Exams such as ECGs, chest X-rays, and D-dimer provide nonspecific findings. However, since almost all cases show at least one risk factor for pulmonary embolism, the careful examination of acquired or hereditary factors is essential. A help in the stratification of risk can come from scoring systems (Wells, Geneva and other), which have shown a good predictive capacity.

\section{Aortic aneurism}

Aneurism of the abdominal aorta (AAA) is another high-risk condition both for its high mortality rate and for the difficulty of 
the diagnosis. ${ }^{11}$ In about half the patients with AAA, the condition is not discovered in the initial diagnosis. Patients with ruptured AAA often have no known history of aneurism. The classic triad of abdominal or back pain, hypotension and pulsating abdominal mass is present only in a minority of cases. Physical examination is not reliable: AAA is frequently not palpable and the status of femoral pulses in not helpful. X-rays are neither sensitive nor specific; they can evidence the calcifications of the aorta only in $60 \%$ of the cases. Ultrasounds are indicated because very sensitive and specific for AAA, but a lot depends on the ability of the examiner, the constitution of the patient and the presence of abdominal gas; furthermore, while ultrasounds can detect the presence of AAA they cannot detect its rupture.

This diagnosis should always be taken into account in the case of abdominal, flank or back pain in patients over 50 with cardiovascular risk factors. When an asymptomatic AAA is incidentally detected, it is extremely important to document that patients have been informed of their condition and instructed to obtain an adequate follow-up. The information must be formally provided also to the patient's healthcare providers.

\section{Conclusions}

The present paper seeks to provide indications concerning the areas were the diagnostic process in the ED can be improved in order to reduce litigation. Further research is necessary to identify more precisely the aspects of the diagnostic process that are most often the subject of malpractice claims and to ascertain which ones are more open to improvement. The present analysis emphasizes the need that ED doctors pay special attention when facing these conditions. One should not forget however that mitigating the risk requires a broader focus because of the many specialists that provide advice or direct medical care in the ED. Therefore changes focused solely on ED doctors risk missing a number of errors and failing to take into account the complex interactions that occur when treating critical patients.

\section{References}

1. Bianchin C. Ridurre il rischio di contenzioso. Decidere in Medicina 2015;5:49-53.

2. Brown TW, McCarthy ML, Kelen GD, Levy F. An epidemiologic study of closed emergency department malpractice claims in a national database of physician malpractice insurers. Acad Emerg Med 2010;17:553-60.

3. Karcz A, Holbrook J, Auerbach BS, et al. Preventability of malpractice claims in Emergency Medicine: a closed claims study. Ann Emerg Med 1990;19:865-73.

4. American College of Emergency Physician. Developed by members of the ACEP Medical Legal Committee. Summary of malpractice claim data $\&$ trends from three sources. An information paper. October 2013.

5. Kachalia A, Ghandi TK, Puopolo AL, et al. Missed and delayed diagnoses in the Emergency Department: a study of closed malpractice claims from 4 liability insurers. Ann Emerg Med 2007;49:196-205.

6. Guly HR. Diagnostic errors in an Accident and Emergency Department. Emerg Med J 2001;18:263-9.

7. Bianchin C, Zanella R. Rischio medico-legale nella gestione delle ferite in Pronto Soccorso. RischioSanità 2013;51:9-12.

8. Geynne A, Barber P, Tavener F. A review of 105 negligence claims against accident and emergency departments. J Accid Emerg Med 1997;14:243-5.

9. Pancioli AM. Advanced stroke care: avoiding medical-legal disasters in the Emergency Department. EMCREG International Monograph from 2009 satellite Symposium in Boston. Adv Standard Care: Cardiovasc Neurovasc Emerg 2010;21-4.

10. Sullivan A, Horejsi TG, Chen S, Feldaman JA. MACEP Risk Management Course. Module 2: Chest Pain. Pulmonary Embolism. Massachussets: Massachusetts College of Emergency Physician; 2011. pp 1-25.

11. Hopkins J, Farrugia L, Suchensky M. MACEP Risk Management Course. Module 1: Abdominal Pain. Abdominal Aortic Aneurysm. Massachussets: Massachusetts College of Emergency Physician; 2011. pp 1-13. 